\title{
Functions Antagonistic to Plasmid Formation by $\lambda \boldsymbol{N}^{-}$Chromosomes
}

\author{
By CHRISTOPHER HADFIELD*† AND WILLIAM J. BRAMMAR \\ Department of Biochemistry, Adrian Building. Unicersity of Leicester, Leicester LEI 7RH, UK
}

(Receired 2 December 1983; revised 24 April 1984)

\begin{abstract}
It was concluded in the preceding paper that $\lambda N^{-} \mathrm{cI}^{-}$genomes probably failed to form plasmids within infected Escherichia coli cells because they leakily express functions that act to destabilize the plasmid state. This prediction was investigated by examining the effect upon plasmidforming ability of the loss of possible anti-plasmid functions. The loss of Ter function was found to allow long-term plasmid formation, although the efficiency of initial plasmid formation and the heritable stability without selection were low. The combined loss of the int, red and gam gene functions also promoted plasmid growth, although the absence of $\mathrm{Ter}^{i}$ was necessary. In contrast, the presence of $\operatorname{Ter}^{80}$ (due to an $h^{80}$ substitution) did not prevent plasmid formation when the $i n t$, red and gam genes were absent, indicating that $\mathrm{Ter}^{80}$ does not attack the closedcircular form of the $\lambda$ chromosome. The combined loss of the ter, int, red and gam gene functions facilitated fully efficient inheritance of the $\lambda N^{-} \mathrm{cI}^{-}$plasmids in the absence of selection, although the efficiency of initial plasmid formation remained low. However, cells harbouring such plasmids suffered a decline in viability, indicating that the plasmids expressed a function (or more than one) that acts to debilitate the host cells - presumably an effect that is increased with this genotype because the modified $\lambda N^{-} \mathrm{Cl}^{-}$plasmids are inherently more stable. The possible involvement of the $\lambda S$ and $k i l$ functions in destabilization is discussed.
\end{abstract}

\section{INTRODLCTION}

Mutants of bacteriophage $\lambda$ lacking $N$ gene function are able to form plasmid replicons within infected Escherichia coli cells (Signer, 1969; Kleckner \& Signer, 1977). However, they do so inefficiently and the plasmid state is very unstable and short-lived (Hadfield \& Brammar, 1984). In the latter paper, we suggested that the cause of these defects in plasmid formation is the $N$-independent leaky expression of certain $\lambda$ functions that act to destabilize the plasmid state. If so, then the elimination of such functions by mutation should enhance plasmid formation and stability.

Candidates for the role of antagonizing plasmid formation are the products of the $\mathrm{int}$, red and gam genes, the $b$-region encoded endonucleases and the packaging functions. The int, red and gam gene products act to modify the structure of the monomeric closed-circular $\lambda$ DNA, which is formed initially upon infection. Potential plasmid destabilization by the packaging system is mediated by the terminase function (Ter), through its binding-cutting action at the cos site and the initiation of DNA encapsidation. Endonucleases encoded by the endo and hen genes are able to cleave both $\lambda$ and $E$. coli DNA.

The endo, ben, int, red and gam genes are all dispensable for lytic phage growth, and are located within a region of the $\lambda$ genome that can readily be deleted or replaced. The terminase is encoded by two ter genes, $A$ and $N u l$, both of which are essential for lytic growth (Wang \& Kaiser, 1973: Becker \& Gold, 1978). Thus, Ter function can be eliminated by available amber mutations in gene $A$, which give a functional $A$ protein in suppressing host cells (to enable lytic phage propagation) but eliminate Ter activity in non-suppressing cells.

+ Present address: Leicester Biocentre. Medical Sciences Building. University of Leicester. Leicester LFI TRH. LK 
In this paper, we report the effects of eliminating the int, red, gam and $A$ gene functions upon plasmid formation by $\lambda N^{-}$phage genomes, and also of the presence of $\phi 80$ left-arm substitutions affecting the endonuclease and terminase genes. Our results show that the plasmid state can be stabilized by the use of appropriate mutant phage genotypes.

\section{METHODS}

Bacterial and phage strains. These are listed in Tables $I$ and 2, respectively.

General techniques. Media, and methods other than those detailed below, were as described by Hadfield \& Brammar (1984).

Analysis of the DNA species present in cells by agarose-gel electrophoresis. Cells from $1 \mathrm{ml}$ overnight-grown liquid culture were pelleted in an Eppendorf microfuge and resuspended in $70 \mu \mathrm{l} 25 \%(\mathrm{w} / \mathrm{v})$ sucrose, $50 \mathrm{~mm}-\mathrm{Tris} / \mathrm{HCl}$

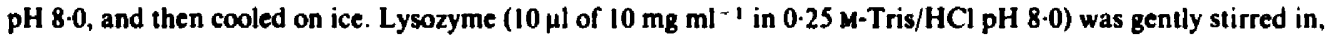
and after $5 \mathrm{~min}$ on ice $20 \mu \mathrm{l} 0.25 \mathrm{M}$-EDTA pH $8.0 \mathrm{was}$ added. After a further $10 \mathrm{~min}$ on ice, $60 \mu \mathrm{l} 2 \%$ (v/v) Triton $X-100,50 \mathrm{~mm}-\mathrm{Tris} / \mathrm{HCl} \mathrm{pH} 8 \cdot 0,65 \mathrm{~mm}-\mathrm{EDTA}$ was gently stirred in, and following a further $5 \mathrm{~min}$ on ice the tube was transferred to a $70^{\circ} \mathrm{C}$ water bath for $5 \mathrm{~min}$ to ensure total lysis. The tubes were then spun at 15000 r.p.m. for $30 \mathrm{~min}$ and the centrifuge stopped without braking. The supernatant was decanted into another Eppendorf tube and gently extracted with 1 vol. phenol (redistilled, water-saturated and equilibrated with $10 \mathrm{~mm}-\mathrm{Tris} / \mathrm{HCl} \mathrm{pH} 8.0$ before use) plus 1 vol. chloroform. After spinning in a microfuge for $5 \mathrm{~min}$, the upper phase was carefully removed with a Pasteur pipette, avoiding the protein debris at the interface. This was re-extracted with phenol/chloroform, the upper phase was transferred to a new tube, and 0.1 vol. sodium acetate pH 5.6 was added, followed by 2 vols absolute ethanol. After gentle mixing, the precipitated DNA (plus RNA) was pelleted by a 10 min spin in a microfuge. The tube was drained and the pellet washed with $70 \%(v / v)$ ethanol. The pellet was vacuum dried and resuspended in $100 \mu \mathrm{l} 10 \mathrm{~mm}-\mathrm{Tris} / \mathrm{HCl}, 1 \mathrm{~mm}-\mathrm{EDTA}, \mathrm{pH} 8 \cdot 0$.

Table 1. E. coli strains

\begin{tabular}{|c|c|c|}
\hline Strain & Genotype & Reference or Construction \\
\hline AA 129 & 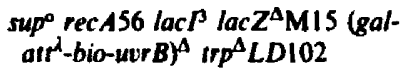 & Anilionis (1977) \\
\hline QR47 & supE & Weil \& Signer (1968) \\
\hline Ymel & sup $F$ & Goldberg \& Howe (1969) \\
\hline C600 & supE tonA thr leu thi & Appleyard (1954) \\
\hline $\mathrm{CH} 28$ & supF tonA & TS selection of Ymel \\
\hline CHSI & $\sup E \operatorname{ton} A$ & T5 selection of QR47 \\
\hline $\mathrm{CH} 13$ & supE recAl IonA & Hadfield \& Brammar (198 \\
\hline CHIS7 & supE tonA thr leu thi (P2) & Lysogen of $\mathrm{C} 600$ \\
\hline
\end{tabular}

Table 2. Phage $\lambda$ strains

$\lambda$ stock no.

$(\mathrm{CH} \#)$

Genotype

Reference or cross

$\lambda^{+}$

$\lambda \operatorname{limm}^{21}$

$\lambda \mathrm{cl}^{20}$

$\lambda i m m^{21} \operatorname{Sam} 7$

$\lambda h^{80} \operatorname{trp51} \mathrm{Nam7,am53}$

גh80 irp50 Nam7,am53 cltsA12

$\lambda$ Aam 32

$\lambda$ Aam32 $\mathrm{imm}^{21} \mathrm{cl}^{-}$

$\lambda h^{\lambda}$ Aam 32 irp50 Nam7,am53 cltsAt2

$\lambda h^{\lambda}$ Aam32 rrpBG2 Nam7,am53 $\mathrm{cI}^{\Lambda}$ KHS4

$\lambda h^{80} \mathrm{atr}^{\lambda} \mathrm{imm}^{21} \mathrm{cl}^{-}$

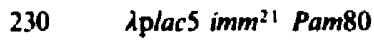

$253 \lambda \lambda h^{i} \operatorname{trp} 50 \mathrm{Nam} 7, a m 53$ cItsAt2

$285 \lambda h^{\lambda}$ Aam32 $\operatorname{trp51} \mathrm{Nam7,am53} \mathrm{cI}^{-}$

$286 \lambda h^{\alpha} \operatorname{irp51} \mathrm{Nam7,am53} \mathrm{cl}^{-}$
Liedke-Kulke \& Kaiser (1967)

Meselson (1964)

W.J.B., unpublished

Brammar et al. (1974)

Franklin (1971)

Wang \& Kaiser (1973)

$\lambda 159 \times \lambda 229$ : clear plaques on $\mathrm{CH} 28$

$\lambda 138 \times \lambda 161$ : selected on CHI57; purified on CHSI

Hadfield (1980): Murray \& Brammar (1973)

N. E. Murray (Edinburgh University). unpublished

R. Pastrana (Edinburgh University), unpublished

$\lambda 138 \times \lambda 6$ : selected on $\mathrm{CH} 157$ : purified on CHSI

$\lambda 58 \times \lambda 164:$ selected on $\mathrm{CH} 131$

$\lambda 58 \times$ 2253: selecied on $\mathrm{CH} 131$ 
Samples $(10 \mu \mathrm{l})$ of this preparation were mixed with an equal volume of $10 \%$ (w/v) Ficoll, $0 \cdot 05 \%$ (w/v) xylene cyanol FF and loaded into a slot of a $1 \%(w / v)$ agarose gel (Tris/acetate buffer $\mathrm{pH} 8 \cdot 2)$ for electrophoresis. Afterwards, the gel was stained by $15 \mathrm{~min}$ immersion in ethidium bromide $\left(2.5 \mu \mathrm{g} \mathrm{ml}{ }^{-1}\right)$, then washed for the same period in distilled water. This after-staining avoided loss of the supercoiled plasmid DNA band during the run due to unwinding caused by the intercalating dye (see Bauer ef al., 1980).

Transfer of DNA from agarose gel to nitrocellulose filter. labelling of probes, hybridization and autoradiography. DNA was bidirectionally transferred from the agarose gel by the method of Smith \& Summers (1980). DNA probes were labelled with 3:P by nick-translation, and the hybridization and autoradiography performed essentially as described by Jeffreys \& Flavell (1977).

Preparation of phage DNA and isolation of restriction fragments. Phage DNA was prepared by Murray at al. (1977). DNA fragments resulting from digestion by restriction enzymes (Bethesda Research Laboratories) were separated at $4{ }^{\circ} \mathrm{C}$ in a horizontal gel of $0.8^{\circ}$ low-gelling-temperature agarose (Miles) in Tris/acetate buffer ( $40 \mathrm{~mm}$-Tris, $20 \mathrm{~mm}$-sodium acetate, $1 \mathrm{~mm}$-EDTA, pH 8.2) containing $10 \mu \mathrm{g}$ ethidium bromide $\mathrm{m}^{-1}$. Bands were cut out of the gel under UV illumination. placed in a $2 \mathrm{ml}$ Eppendorf tube with 3 vols $8 \mathrm{M}$-sodium perchlorate (precleaned by passing through a glass-fibre filter) and heated for $5 \mathrm{~min}$ at $65^{\circ} \mathrm{C}$ to melt the gel. Afterwards I mg Kieselgel $\mathbf{H 6 0}$ (Merck product 7736) was added for every $200 \mu \mathrm{l}$, mixed and the mixture left for 10 min at room temperature. The DNA-bound Kieselgel was pelleted by a $2 \mathrm{~min}$ spin in a horizontal microfuge and the supernatant discarded. The pellet was washed by dispersing in 3 vols $6 \mathrm{~m}$-sodium perchlorate (pretreated as above), repelleting and then draining off the supernatant. DNA was eluted from the Kieselgel by dispersing in $200 \mu \mathrm{l}$ TE (10 mM-Tris/HCl pH 8. I mM-EDTA) and pelleting the Kieselgel. The pellet was re-extracted with $100 \mu \mathrm{l}$ TE and the supernatants were pooled.

The DNA was cleaned by precipitation, by addition of $33 \mu \mathrm{l} 2 \mathrm{M}$-sodium acetate pH 5.6 and $667 \mu \mathrm{l} \mathrm{ethanol}$. After a 15 min spin in a microfuge, the DNA pellet was washed with $70 \%$ ethanol, vacuum dried and resuspended in $300 \mu \mathrm{l} \mathrm{TE}$. Residual carbohydrate contamination was removed by adding $300 \mu \mathrm{l} \mathrm{2.5} \mathrm{M}$-potassium phosphate pH 8.0 and $300 \mu \mathrm{l}$ methoxyethanol, and mixing. After spinning for $5 \mathrm{~min}$ in a microfuge, the upper phase was removed to a new tube and gently mixed with an equal volume of ethanol. After a 5 min spin in the microfuge, the DNA resided on the surface of a droplet of phosphate buffer at the bottom of the tube. This droplet was removed with a pipette and the rest of the solution drained off, leaving the DNA in the tube. The DNA was then washed with $70 \%$ ethanol and any further phosphate droplets were removed in the same way. After a third $70^{\circ}$ ethanol wash (no droplets should be present or else a further wash is necessary), the DNA was drained free of ethanol, vacuum dried and then resuspended in $50 \mu \mathrm{l} \mathrm{TE}$.

\section{RESULTS}

\section{Choice and construction of phages}

The choice of $\lambda N^{-}$transducing phages was governed by several factors. First was the requirement to have phages of the same overall genetic composition, barring those differences that were specifically required for individual experiments. Secondly, the red $\mathrm{gam}^{-}$phage employed must also be able to propagate adequately by lytic growth. Efficient lytic growth on a $\mathrm{rec}^{+}$host (also sup ${ }^{+}$due to the need to suppress amber mutations) by such a phage requires the presence of a chi site (Enquist \& Skalka, 1973; McMilin et al., 1974; Henderson \& Weil, 1975). It was also required to be able to make Aam mutants. Use of $h^{80}$ provided a convenient counterselectable marker for the introduction of $h^{\lambda}$, particularly so when Aam was to be introduced, since the $N$ gene also contained amber mutations. $h^{80}$ is also of interest, as well as a genetical tool, since it encodes the packaging functions of the related lambdoid phage $\phi 80$. Phages $\lambda$ and $\phi 80$ package their DNA in a similar manner, and their terminases perform the same functions. The $\phi 80$ and $\lambda$ terminases are encoded by equivalent genes and are composed of similar subunits (Sumner-Smith \& Becker, 1981; Sumner-Smith \& Gold, 1981). The terminal sequences of the two phages are the same and the terminases are able to cross-react (Murray et al., 1975). Other leakily expressed products of genes contained within the $h^{i}$ and $h^{80}$ regions (extending from $\cos _{\mathbf{l}}$. to att) may affect plasmid formation differently. Thus, the $\lambda$-encoded endonucleases may differ in activity from similar $\phi 80$ endonucleases. Differences due to gene content may also occur. Although the gene content of $\lambda$ and $\phi 80$ is similar (Youderain, 1977), the $\phi 80$ genome is $8 \%$ smaller than that of $\lambda$, indicating the possible absence of some genes that are not essential for growth.

The $\lambda N^{-}$transducing phages chosen were $\lambda h^{80} \operatorname{trp} 51 \mathrm{Nam} 7, a m 53 \mathrm{cl}^{-}$and $\lambda h^{80} \operatorname{trp} 50 \mathrm{Nam} 7$. am53 cltsAt2. Both phages contain the trpED genes, the leader sequence, the attenuator and the 

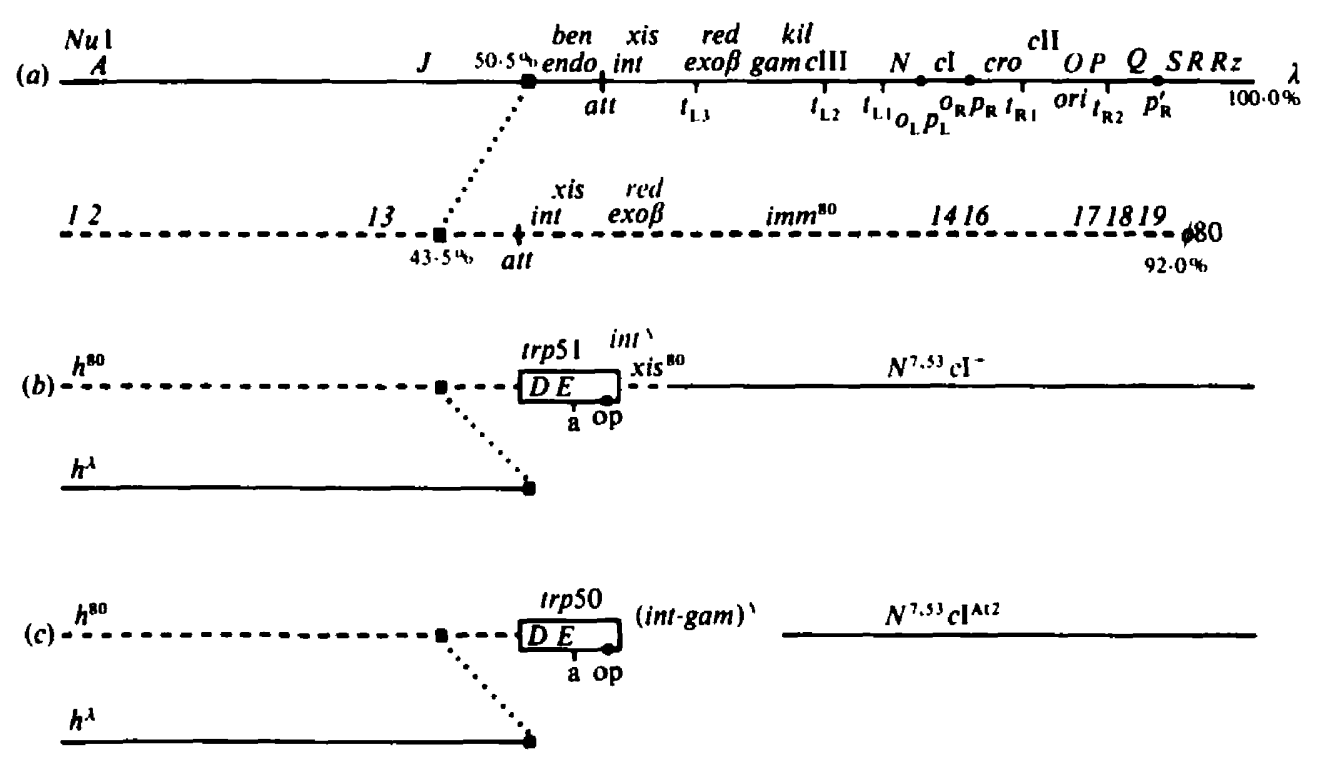

Fig. 1. Structures of trp-transducing phage genomes used to monitor $\lambda N^{-}$plasmid formation (not to scale). (a) wild-type $\lambda$ and $\phi 80$, showing the region of homology to the left of att (Fiandt et al., 1971): (h) $\lambda h^{80} \operatorname{trp51} \mathrm{Nam7}, a m 53 \mathrm{cl}^{-}$, showing the $h^{i}$ substitution; (c) $\lambda h^{80} \operatorname{trp50} \mathrm{Nam} 7, a m 53 \mathrm{cl} / s \mathrm{Al} 2$, showing the $h^{i}$ substitution.

operator-promoter; the latter enables independently controlled expression, so there is no reliance upon plasmid-initiated transcription. The $\operatorname{trp} E$ and $D$ genes both contain chi sites, as do the $h^{80}$ and $a t t^{80}$ regions (Anilionis, 1977). The trp5l substitution is associated with a deletion of the int gene (Brammar et al., 1974), and this $\lambda h^{80} N^{-}$phage is known to be almost totally defective for plasmid formation (Hadfield \& Brammar, 1984). The trp50 substitution is associated with a bigger deletion, extending from $a t t$, through int and red, up to and including gam, but not beyond (Franklin, 1971). The two phages therefore provide for a direct comparison of the effect of the combined loss of int, red and gam on $\lambda N^{-}$plasmid formation. Derivatives carrying $h^{\dot{\lambda}} A^{\bullet}$ and $h^{\dot{\lambda}} A a m 32$ were constructed for both of these transducing phages, as described in Table 2. The Aam 32 allele used was found by Wang \& Kaiser (1973) to eliminate Ter function in a non-suppressing host. The structures of these phages are shown in Fig. 1.

\section{Effects of the loss of the int, red and gam genes on $\lambda h^{80} \mathrm{~N}^{-}$plasmid formation}

The sup recA trpED $D^{\Lambda}$ transduction host cells (AA129) were infected over a range of

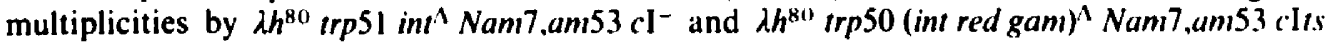
and transductants selected on tryptophan-free plates at $37^{\circ} \mathrm{C}$. This temperature inactivated the thermolabile repressor of cltsAt2. Table $3(a, b)$ shows a dramatic difference in transduction performance between the two genotypes. The $\lambda h^{80} \operatorname{trp} 51 \mathrm{int} t^{\Delta} \mathrm{Nam} 7, a \mathrm{~m} 53 \mathrm{Cl}^{-}$control showed very little transduction, and the transduced colonies formed failed to grow beyond $0.5 \mathrm{~mm}$ in diameter, as found before. In contrast, $\lambda h^{80}$ trp 50 (int red gam ${ }^{\Delta} \mathrm{Nam} 7, a m 53 \mathrm{cl}$ ts transduced at a frequency of $22 \%$ when singly infecting and with complete efficiency at an m.o.i. of 6 , although the efficiency dropped at higher m.o.i., indicating an inherent defect in the plasmids. However, the cells transduced by $\lambda h^{80} \operatorname{trp50}$ (int red gam) ${ }^{4} \mathrm{Nam} 7, \mathrm{am} 53 \mathrm{cl} / s$ continued to grow and formed large colonies on the selective plates, indicating continued variability of the plasmids when established.

Transductant isolates were tested for the heritable stability of the plasmids by picking a purified colony from a selective plate into $5 \mathrm{ml} \mathrm{L}$ broth containing tryptophan and growing overnight to saturation (about 15 generations of growth). Retention of the plasmid was measured by diluting the cultures in bacterial buffer and plating equal numbers of cells on 
tryptophan-free (TRP-) and tryptophan-containing (TRP ${ }^{+}$) plates. The maintenance stability value was given by the percentage ratio of the number of colonies that grew on TRP - plates over those on TRP ${ }^{+}$plates. The plasmids were well maintained, again indicating good viability of this genotype. A stability value of $91^{\circ}$ was observed for singly-infected cells, and similar values for multiply-infected cells, showing that the number of input phages did not influence plasmid maintenance (Table $3 b$ ). When transduced cells were grown up in TRP- liquid medium, the resulting cultures showed no evidence of cell lysis. This contrasts with the selected $\lambda N^{-}$tir transductants previously examined, of which selected cultures contained a proportion of lysed cells (Hadfield \& Brammar, 1984). Presumably, leaky expression of the lysis proteins by the $\lambda h^{311}$ trp50 (int red gam) ${ }^{\Lambda} \mathrm{Nam} 7, a m 53 \mathrm{cl}$ ts plasmids was low enough to be tolerated by the host cells.

The presence of intracellular $\lambda$ genomes was examined by making lawns of transduced cells and spot-testing. All 20 isolates tested showed the same responses: (1) they allowed the growth of $\lambda$ imm $^{21}$; (2) $\lambda$ imm ${ }^{21}$ Pam80 produced occasional plaques, indicating either $P$ product complementation or marker rescue of a functional $P^{+}$allele from intracellular $\lambda$ genomes: (3) $\lambda \mathrm{imm}^{21} \mathrm{Sam} 7$ also grew, giving a confluent spot, presumably due to complementation involving activation of the plasmids $p_{\mathrm{R}}^{\prime}$ promoter by the $Q$ gene product of the superinfecting phage: (4) $\lambda \mathrm{CI}^{-}$grew, indicating that the transduced cells were not fully anti-immune : (5) $\lambda c \mathrm{I}^{+} \mathrm{grew}$ to form a turbid spot, showing that there was too little cro protein within the carrier cells to inhibit $c$ lrepressor synthesis.

Thus, although $\lambda h^{80} \operatorname{trp} 50$ (int red gam $)^{4} \mathrm{Nam} 7, a m 53 \mathrm{clts}$ had greatly improved plasmid characteristics, it still had the flaws of lower than maximal initial plasmid formation and maintenance in non-selecting conditions. Lack of anti-immunity of the carrier cells may also indicate less than maximal plasmid copy number. Such defects might be due to an inhibitory function, such as the action of Ter.

\section{Effects of the loss of the int, red and gam genes on $\lambda h^{\wedge} N^{-}$plasmid formation}

Plasmid formation by $\lambda h^{i} \operatorname{trp} 50$ (int red gam) $)^{\prime} \mathrm{Nam} 7, a m 53 \mathrm{cIts}$ was compared with that by

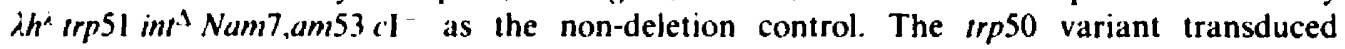
exceedingly rarely when infecting singly, producing very tiny colonies (about $0.2 \mathrm{~mm}$ in diameter) that remained small and could not be sub-cultured. The results otherwise showed complete failure to transduce in the plasmid mode by either genotype (Table $3 c, d$ ). From these results it would seem that the success of $\lambda h^{80} \operatorname{trp} 50$ (im red $\left.\mathrm{gam}\right)^{\Lambda} \mathrm{Nam} 7$, am $53 \mathrm{cIts}$ can be attributed to the combined presence of the $h^{81}$ left-arm substitution and the int-red-gam deletion. This suggests that the $\phi 80$ terminase, unlike that of $\lambda$, is unable to attack and destabilize the monomeric closed-circular plasmid form of the $\lambda$ chromosome.

\section{Effects of the loss of Ter function on $\lambda h^{\prime} N$ plasmid formation}

The effects of Ter function on $\lambda N$ plasmid formation were examined by contrasting plasmidforming ability when the enzyme was present and absent. Initially this was done with the red and gam genes present. Thus, $\lambda h^{\circ}$ Aam $32 \mathrm{trp} 51 \mathrm{im} \mathrm{t}^{\circ} \mathrm{Nam} 7, a \mathrm{~m} 53 \mathrm{Cl}$ was examined for Ter plasmid formation, with the $\lambda h^{\prime} A^{+} t r p 51$ int ${ }^{i} N a m 7, a m 53 \mathrm{Cl}^{-}$variant tested previously (Table $3 c$ ) serving as the $\mathrm{Ter}^{*}$ control. With active $\mathrm{Ter}^{\wedge}$ present the phage completely failed to transduce in the plasmid mode. In contrast, the Ter"-defective Aam variant transduced 5-6 ${ }_{0}^{\circ}$ of the infected cells when infecting singly and more when infecting multiply, reaching a maximum of $14.5^{\circ}$. at an m.o.i. of 6 (Table $3 \ell$ ). These transduced cells continued to grow, forming progressively larger colonies, indicating the continued viability of the established plasmids. The ter plasmids were also quite well maintained during non-selecting growth, at a level of around $10^{\circ}$ in the stability test. From these results it is clear that $\mathrm{Ter}^{\prime}$ has a highly effective antiplasmid activity that can prevent $\lambda N^{-}$plasmid formation, both when the DNA is circular monomeric (as with $\lambda h^{\prime} \operatorname{trp} 50 \mathrm{Nam} 7, a m 53 \mathrm{clts}$ ) and when it is concatenated (as apparent with $\lambda h^{2}$ trp5I $\mathrm{Nam} 7 . a m 53 \mathrm{CI}^{-}$)

Absence of Ter activity is therefore essential for successful plasmid formation by $\lambda h^{\star} N$ genomes. This supports the view that the benignity of $h^{80}$ to plasmid formation in the absence of 
Table 3. Effects of red and gam, of host range and of Ter function on the establishment and stability of $\lambda$ plasmids

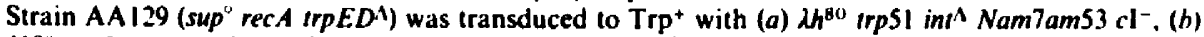

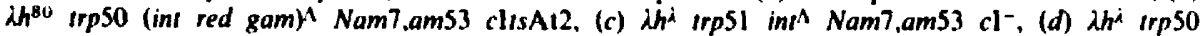

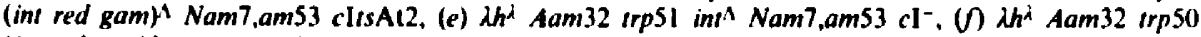
(int red gam) ${ }^{4} \mathrm{Nam} 7, a m 53$ clisAt2. Both the frequency of transduction and the maintenance stability figures are mean values for four independent measurements at each m.o.i. Variation between the independent measurements on either side of the mean was within one-tenth of the mean value for figures over $5 \%$ and within one-fifth of the mean for figures under $5 \%$.

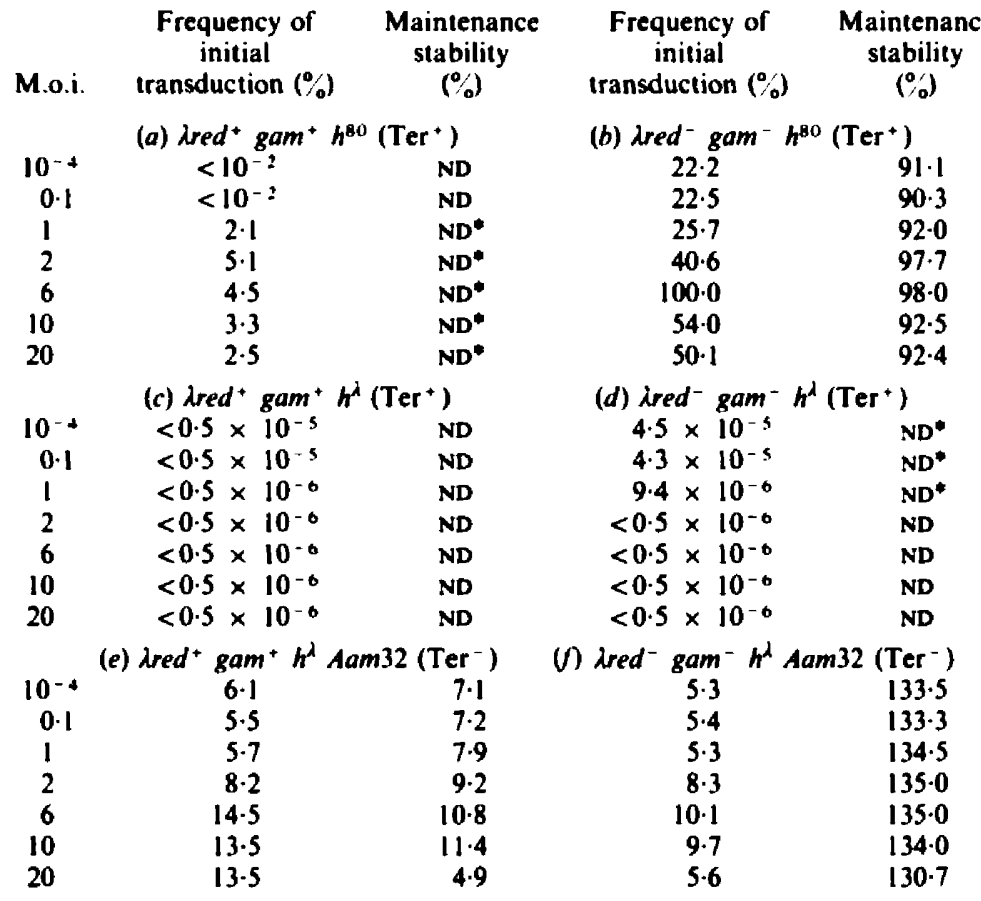

ND, Not done due to failure to obtain transductants; ND*, not done due to failure of transductants to subculture.

the int, red and gam genes, but not in their presence, is due to failure of $\operatorname{Ter}^{80}$ to attack monomeric circular forms of the $\lambda$ genome. Although absence of Ter function led to improved plasmid-forming ability, the inability to transduce with complete efficiency and to be maintained completely within the cells during growth without selection indicates that other defective characteristics remain, as is known to be the case. Several of the transduced isolates were spottested for the presence of intracellular $\lambda$ genomes and for anti-immunity, as described for the $\lambda h^{80}$ trp50 Nam7,am53 cIts transductants. Intracellular $\lambda$ genomes were detected, but the cells were not anti-immune. Thus, plasmid numbers were not apparently being controlled by cro protein autorepression.

\section{Effects on $\lambda h^{\lambda} N^{-}$plasmid formation of the combined loss of Ter and the int-red-gam gene functions}

The combination of a ter deficiency with an int-red-gam deletion eliminates two proven sources of $\lambda$ plasmid instability. The question remains as to whether these functions are the only ones that destabilize the plasmid. Analysis of a $\lambda N^{-}$phage carrying both of these alterations was undertaken to reveal if other functions remain to prevent non-defective plasmid formation and growth. Thus the phage $\lambda h^{\lambda} A a m 32$ trp50 (int red gam) ${ }^{\Delta} N a m 7, a m 53$ cIts was tested for plasmid formation, in comparison with the similar $\lambda h^{\lambda} A^{+}$trp50 (int red gam $)^{\Delta} \mathrm{Nam} 7$, am 53 clts variant tested previously (Table $3 d$ ), which was totally defective, and the $\lambda h^{\lambda} A a m 32 \operatorname{trp} 51$ int $t^{\Delta}$ 


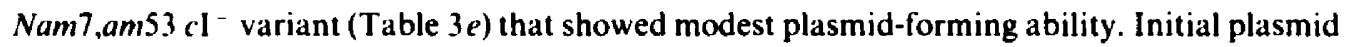
formation by $\lambda h^{i} \mathrm{Aam} 32 \mathrm{trp} 50 \mathrm{Nam} 7, a \mathrm{~m} 53 \mathrm{cl} t \mathrm{~s}$ was at a similar frequency to that found with the $\lambda h^{i} \operatorname{Aam} 32 \operatorname{trp} 51 \mathrm{Nam} 7, a m 53 \mathrm{cl}^{-}$variant (Table $3 \mathrm{f}$. The transduced colonies kept growing. and selected liquid cultures showed no evident cell lysis. However, the colonies were small: approximately $0.2 \mathrm{~mm}$ in diameter after $24 \mathrm{~h}$ incubation at $37 \mathrm{C}$. compared with about $2.0 \mathrm{~mm}$ for uninfected cells or cells transduced by other $\lambda$ plasmids.

Plasmid maintenance stability values showed a change with the $\lambda h^{\wedge} A a m 32 \operatorname{trp} 50$ (int red $\mathrm{gam})^{\Lambda} \mathrm{Nam} 7, \mathrm{am} 53 \mathrm{cl} / \mathrm{s}$ genotype. Values of over $130^{\circ}$, were found, independent of the initial m.o.i. Since the same numbers of cells were plated on both the TRP- ${ }^{-}$and TRP ${ }^{+}$plates for these stability tests, it must be interpreted that fewer were able to grow on the non-selecting plates after growth in non-selecting liquid medium. Thus selection for growth with tryptophan supplied endogenously by the enzymes encoded on the plasmids evidently enabled greater survival of the transduced cells than when the tryptophan was supplied externally.

The colonies obtained after growth in TRP ${ }^{+}$broth, on both the TRP ${ }^{-}$and TRP ${ }^{+}$plates, were of two distinguishable sizes: either $\sim 2 \mathrm{~mm}$ or $\sim 0.2 \mathrm{~mm}$ in diameter after $24 \mathrm{~h}$ incubation at $37^{\circ} \mathrm{C}$. When colonies were picked from TRP ${ }^{-}$plates on to fresh TRP ${ }^{+}$plates, three types of growth response were obtained. The larger colonies either continued to grow well or grew only slightly. The small colonies either grew slightly or failed to grow at all. When those colonies that grew were picked again on to TRP ${ }^{+}$plates a further decrease in ability to grow was observed. This seems to indicate that the viability of the plasmid-carrying cells progressively declines. However, contrarily, this measurable decline in individual colony viability did not coincide with a demise of the transduced population as a whole, since liquid cultures of the plasmid-carrier cells could be grown up to normal stationary phase density in both selecting and non-selecting medium. The phenomenon that the viability of transduced cells is greater under plasmid-selective conditions than in non-selecting conditions cannot be explained from the data available here. However, it does seem that the $\lambda h^{\lambda}$ Aam $32 \operatorname{trp50}(\text { int red gam })^{4} \mathrm{Nam} 7$, am $53 \mathrm{cIts}$ plasmids express a protein that debilitates the host cells in some way, without affecting the plasmids themselves. Spot-tests on transduced cells gave the same response as described earlier for the $\lambda h^{80} \operatorname{trp} 50 \mathrm{Nam7}$, am $53 \mathrm{clts}$ variant. Again failure of the cells to display anti-immunity indicated that the intracellular $\lambda$ genomes were probably not present at a maximal copy number controlled by cro protein autorepression.

\section{Confirmation that the modified $\lambda N^{-}$genomes transduce by forming plasmids}

The host-phage system employed in this study was designed so that transduction could only occur via plasmid-forming $\lambda$ genomes (Hadfield \& Brammar, 1984). The lack of complete heritable stability of a transducing marker, as found in two cases, accords with its being plasmidborne. Verification of the plasmid nature of the transducing $\lambda$ genomes was sought by analysis of the DNA species within transduced cells.

Total DNA was prepared from transduced cells and compared with that from uninfected cells by agarose-gel electrophoresis. Only one DNA band was obtained from the uninfected cells, whereas a second band was also obtained from the transduced cells (Fig. 2a). The int-red-gam deficient phage genomes each formed bands of equivalent mobility to a closed-circular $\lambda$ genome. However, in the cells transduced by $\lambda h^{\lambda} \mathrm{Aam} 32 \operatorname{trp} 51 \mathrm{Nam} 7, a \mathrm{~m} 53 \mathrm{cl}^{-}$this additional band ran slightly faster, more towards the linear configuration, presumably as a consequence of the $\mathrm{red}^{+} \mathrm{gam}^{+}$phenotype. The DNAs were prepared from equal volumes of overnightgrown cells. Although cell numbers were similar, the cells gave rise to different amounts of plasmid DNA, dependent upon genotype: $\lambda h^{80} \operatorname{trp} 50 \mathrm{Nam7}$,am53 $\mathrm{cl} t s>\lambda h^{i}$ Aam32 trp5I

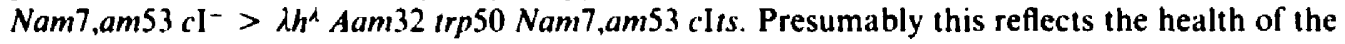
plasmid and the plasmid-host relationship.

Confirmation that these bands result from extrachromosomal $\lambda$ genomes was obtained by hybridizing one copy of a bitransfer of this gel with a ${ }^{32}$ P-labelled $\lambda$ fragment probe containing a segment of the DNA present in each of the transducing $\lambda t r p N^{-}$genomes but having no homology with cryptic $\lambda$ prophage sequences present in the host cell's chromosome (Kaiser \& Murray, 

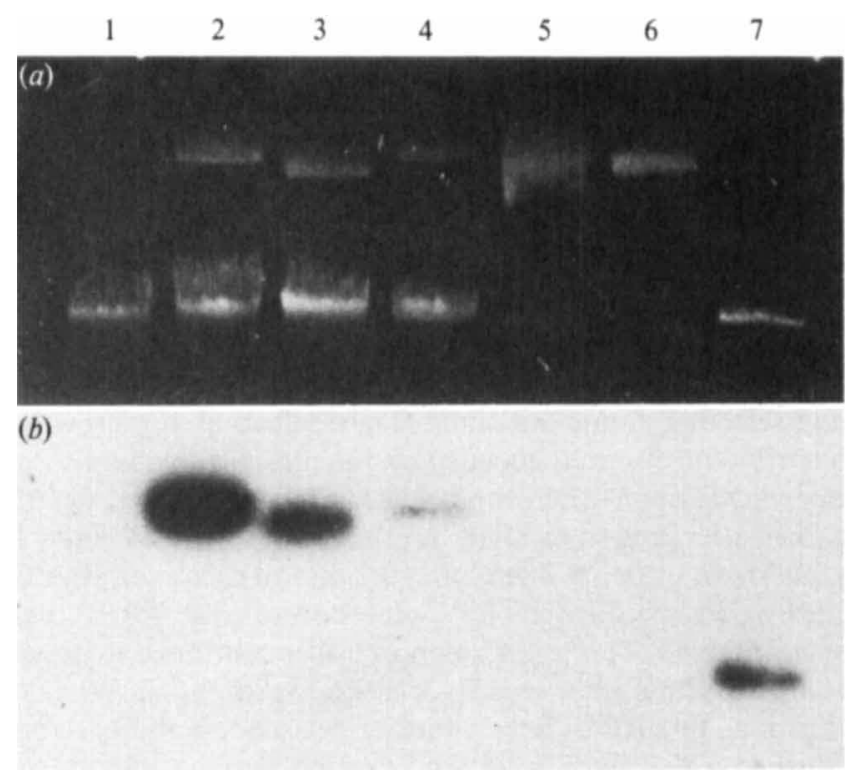

(c)

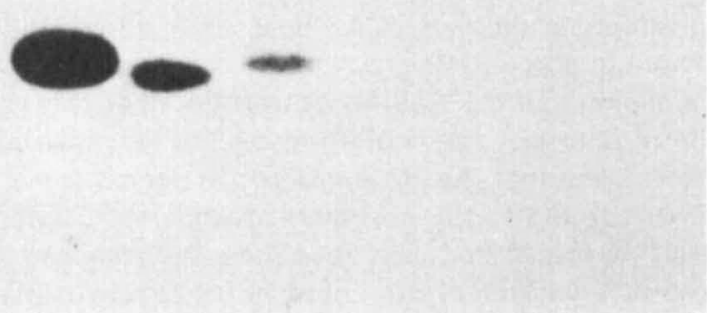

Fig. 2. Characterization of plasmid DNA. (a) Agarose-gel electrophoresis of the DNA from lysed sup" rec $A$ trpED $D^{\Lambda}$ cells (AA 129) transduced to Trp ${ }^{+}$growth by $\lambda$ rep $N^{-}$phages. (1) Non-transduced host control (grown in TRP+ medium): (2) transduced by $\lambda h^{\mathrm{RO}}$ trp50 Nam7,am53 cItsA12: (3) transduced by

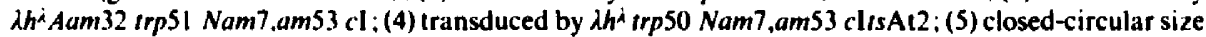
marker, plasmid RP4 (55 kb); (6) $\lambda$-sized closed-circular size marker, cosmid clone $\mathrm{cm}$. a47 (about $49 \mathrm{~kb}$ : Pirrotta et al. 1983); (7) linear $\lambda$ DNA extracted from phage particles (48.5 kb: Sanger $e t a l$. . 1982).

(b) Autoradiograph of one filter copy of a bitransfer of the above gel after hybridization with a $3: \mathrm{P}$. labelled $\lambda$ fragment probe having no homology with the host bacterial chromosome but present in the $\lambda$ transducing phage genomes. Because the $\lambda$ genome has extensive homology with the $E$. coli $\mathrm{K} 12$ chromosome, the $\lambda$ fragment probe used had to be carefully selected. Thus, restriction fragments of $\lambda$ DNA were separated by agarose-gel electrophoresis, Southern transferred to a nitrocellulose filter and then hybridized with "'P nick-translated AA129 host bacterial DNA. Those $\lambda$ fragments having no homology with the bacterial DNA probe failed to give signals after autoradiography. One such fragment. generated by $E c o R I$ cleavage at $65.6^{\circ} \% \lambda^{+}$and $B a m H I$ cleavage at $71.0^{\circ} \% \lambda^{+}$, was used as the probe for autoradiograph (b). It hybridized to each of the $\lambda$ plasmid bands and the linear $\lambda$ DNA control. (c) Autoradiograph of the second copy filter after hybridization with a ":P-labelled $r p E$ fragment probe. This fragment $(2.0 \mathrm{~kb})$ was cleaved from $\lambda t r p B G 2$ ( $\lambda \mathrm{CH} 187)$ phage DNA by restriction at the HindIIl site in $\operatorname{rpD}$ (Hopkins et al. 1976) and the BamHI site in the $\lambda$ DNA at $71.0^{\circ} \lambda^{+}$. The $\operatorname{rrpBG} 2$ substitution ends very close to this BamHI site, so that no homology with $\lambda$ is detectable by hybridization. The trpE fragment probe only hybridized with the trp-transducing $\lambda$ plasmid bands in autoradiograph (c).

1979). Autoradiography revealed hybridization to the additional bands in the transduced cells and not to the host chromosomal DNA (Fig. $2 b$ ). Because the probe fragment used crosses the att-gam deletion breakpoint associated with trp50, signals with this genotype are weaker. This is only noticeable with the smaller amount of $2 h^{i}$ Aam $32 \operatorname{trp} 50 \mathrm{Nam} 7, a m 53 \mathrm{clts}$ DNA, since the signal from $\lambda h^{* 0} \operatorname{trp} 50 \mathrm{Nam} 7, a m 53 \mathrm{cl} t s$ is strong enough to saturate the $\mathrm{X}$-ray film response. The 
second filter copy was hybridized with a trpE fragment probe, which comprised a segment of the trp operon present in the infecting $\lambda N^{-}$transducing phage genomes, but wholly absent in the trpLD102 deletion present in the host cells chromosomes. Autoradiography showed hybridization to the $\lambda$ plasmid bands, and none to the chromosomal DNA, nor the linear phage $\lambda$ DNA (Fig. 2c). This degree of hybridization is sufficient to detect homologous sequences present in the $E$. coli chromosomal DNA, indicating that the transducing genes are probably present only on the plasmids and have not been transferred to the bacterial chromosomes.

\section{DISCUSSION}

The elimination of certain gene functions promoted plasmid formation by $\lambda N^{-} c I^{-}$genomes. The genes involved must have been expressed, and their products acted in some way counter to the plasmid state, for their loss to have caused an improvement in plasmid-forming ability. However, because there are no other promoters, the only way that these genes could have been expressed in $N^{-}$conditions is for some of the transcripts initiated from $p_{1}$ and $p_{R}$ to have leaked through the serially arranged Rho-dependent terminators $t_{L_{1}}-t_{L_{2}}$ and $t_{R_{1}}-t_{R_{2}}$, respectively. These results are therefore in accordance with our finding that substantial leaky transcription of the whole $\lambda$ genome occurs in $N^{-}$conditions (Hadfield \& Brammar, 1984). They also corroborate the proposal that the products of some genes so expressed act to destroy the plasmid form of the $\lambda N^{-}$chromosome initially established upon infection of the host bacterial cell, and thereby make plasmid growth only transient.

The Ter function of $\lambda$ was found to be a powerful anti-plasmid agent, since a further elimination of Ter ${ }^{\lambda}$ activity alone was sufficient to facilitate long-term plasmid formation by $\lambda N^{-} c l$ genomes. Since the red and gam genes were present in this case, the intracellular forms of the $\lambda t e r^{-} N^{-} \mathrm{cl}^{-}$plasmids were presumably not closed-circular, but to some degree polymeric and rolling-circular.

When the red and gam genes were also removed (the int gene was absent in both cases), newly formed plasmids were trapped in the monomeric closed-circular configuration within the rec $A$ host cells. Consequently the inheritance of the plasmids in the absence of selection was substantially increased, such that no plasmid-free segregants could be found. The efficiency of initial plasmid formation was not altered, remaining at the $5 \%$ level. It would seem therefore that if the $\lambda$ plasmids are kept in the closed-circular monomeric form, their ability to propagate through subsequent generations is substantially enhanced - presumably because monomers are produced at the maximum possible copy number. However, some further defect must still remain on the $\lambda \mathrm{ter}^{-} \mathrm{int}^{-} \mathrm{red}^{-} \mathrm{gam}^{-} \mathrm{N}^{-} \mathrm{Cl}^{-}$chromosomes, which prevents them from establishing as plasmids with complete efficiency when they infect potential host cells.

$\lambda N^{-} \mathrm{cl}^{-}$plasmids carrying the $h^{80}$ left-arm substitution failed to form plasmids in the presence of the red and gam genes (int was absent), but did succeed when the int, red and gam genes were all absent. This suggests that for some reason, unlike $\operatorname{Ter}^{\lambda}$, $\operatorname{Ter}^{80}$ does not attack closed-circular $\lambda$ DNA monomers and by its binding-cutting action prevent them from growing as plasmids. In contrast, the polymeric and rolling-circular forms of the genome are susceptible to attack by $\mathrm{Ter}^{80}$.

The failure of the $\lambda \mathrm{rer}^{-} \mathrm{int}^{-} \mathrm{red}^{-} \mathrm{gam}^{-} \mathrm{N}^{-} \mathrm{Cl}^{-}$genomes to establish as plasmids with complete efficiency could be due to the presence of a further gene which codes for still another function with an anti-plasmid activity. In this respect. one previously considered class of genes encoding potential anti-plasmid functions has not been accounted for on these modified $\lambda$ chromosomes. These are the $\lambda$-encoded endonucleases. However, none of the phages used here contained the known endonuclease genes, end $\sigma^{\wedge}$ and $b e n^{i}$, since they were initially $h^{80}$, and the $h^{\prime}$ variants were made by crossing through the region of homology to the left of endo and $b e n^{\wedge}$. However, analogous endo ${ }^{811}$ and ben $^{80}$ genes could be present in all of these variants. Presumably, such endonuclease genes would be transcribed, just like the other genes, although if they were translated and processed into active proteins, they would indiscriminately cleave both plasmid and chromosomal DNA, and, because of the effect of the latter, be a very efficient cause 
of lethality. This suggests that the activities of these endonucleases must be prevented in some way; otherwise $\lambda$ plasmid formation (and also lytic growth) would not be possible. Indeed, the nature of the remaining plasmid defect does not suggest that it is caused by leaky endonuclease expression. Consequently, the low efficiency of initial plasmid formation is probably due to the effect of some other function.

Host bacterial cells harbouring the $\lambda t e r^{-}$int $^{-} \mathrm{red}^{-} \mathrm{gam}^{-} \mathrm{N}^{-} \mathrm{cl}^{-}$plasmids suffered a decline in viability. This defect could result from the leaky expression of one or more functions by these $\lambda$ plasmids that act to debilitate the host cells. The effect of such functions would presumably have become more evident now, and not before because these plasmids are more inherently stable due to their modified genotype. Because the $\lambda$ genome has been extensively characterized, it is possible to predict that certain genes might code for functions harmful to the host cells. Thus, an examination of the $\lambda$ genotype (Echols \& Murialdo, 1978; Szybalski \& Szybalski, 1979) revealed two such genes, $S$ and $k i l$.

The $S$ gene product is involved in cell lysis; its role appears to be to alter the structure of the cytoplasmic membrane so that the lysozymes (products of the $R$ and $R z$ genes) can gain access to the cell wall. Lack of $S$ function in a lytic infection therefore keeps the cell intact and DNA synthesis continues beyond the normal lysis period, leading to over-production of the number of phage particles. Since the cytoplasmic membrane houses many important metabolic functions, any attack upon it may impair the growth of the cell. The $S$ product directly attacks the membrane by hydrolysing phospholipids, leading to the cessation of respiration, alteration of permeability of the cytoplasmic membrane and reduced DNA synthesis (Adhya et al., 1971: Reader \& Siminovitch, $1971 a, b)$. The effects of $\mathrm{p} S$ are not manifest until very late in a normal lytic infection (after about $50 \mathrm{~min}$ ), indicating a tolerance to initial low levels of the protein. This correlates with our previous observations (Hadfield \& Brammar, 1984) that the majority of $\lambda N^{-} r 2, r 1, t 3$ plasmid-containing cells could tolerate the high-level leaky expression of the lysis genes. Thus, expression of $\mathrm{p} S$ may be incapacitating to the cell, but not necessarily lethal, unless in very high amounts.

Induction of $\lambda N^{+} O^{-} P^{-}$lysogens results in killing of the host cells. Such killing cannot be due to replication of the prophage, and has been ascribed to the synthesis of a lethal product by the prophage. The gene $(k i l)$ that codes for this product has been mapped between the gam and $c I I I$ genes (Greer, 1975a). The $k i l$ product (Kil) can be purified as a predominant polypeptide from $\lambda$ infected cells (Greer \& Ausubel, 1979). The killing of induced $\lambda N^{+} O^{-} P^{-}$lysogenic cells is actually characterized by a gradual decline in colony-forming ability, resulting ultimately in the survival of only 1 in $10^{4}$ cells. Such a decrease in cell viability would seem to be similar to that noted above with the $\lambda$ plasmid-carrying cells. How the killing function is mediated is not yet understood, although its action causes cell filamentation (Greer, 1975a, h), which is normally associated with reduced DNA replication (reviewed by Witkin, 1976); yet it does not inhibit DNA synthesis, nor promote the degradation of DNA. Gross RNA and protein synthesis do not seem to be affected either. Certain observations suggest that Kil could have a nuclease activity, in apparent contradiction to the lack of DNA degradation. Other properties of Kil-affected cells suggest that the protein acts on the cell envelope (Greer, 1975a, b).

The activities of the $k i l$ and $S$ proteins could account for some of the defective characteristics of the $\lambda l e \mathrm{r}^{-} \mathrm{int}^{-} \mathrm{red} \mathrm{gam}^{-} \mathrm{N}^{-} \mathrm{Cl}^{-}$plasmids. Clearly the decline in viability of the plasmidcarrying cells could be due to leaky expression of the $k i l$ and $S$ gene products. Whether the expression of such anti-host functions could also cause the low efficiency of initial plasmid formation is not clear. The infected cells that failed to become transduced survived the infection and could grow in non-selecting medium, so the failure of plasmid transduction was not due to lethality. However, it is feasible that plasmid-encoded functions could arrest the growth of a host cell in selecting conditions, and thereby prevent successful plasmid transduction in the majority of cases. An examination of the effects of the loss of $k i l$ and $S$ gene functions should clarify the extent of their contributions to $\lambda$ plasmid defectiveness.

C. H. was supported for this work by a post-graduate training studentship from the Medical Research Council. Our thanks to those who generously provided us with strains. 


\section{REFERENCES}

Adhya, S., Sen, A. \& Mitra, S. (1971). The role of gene $S$. In The Bacteriophage Lambda, pp. 743746. Edited by A. D. Hershey. New York: Cold Spring Harbor Laboratory.

ANILIONIS, A. (1977). A restriction map of Escherichia coli irp operon DNA. PhD thesis, University of Edinburgh.

APPLEYARD, R. K. (1954). Segregation of new lysogenic types during growth of a doubly lysogenic strain derived from Escherichia coli K 12. Genetics 39. 440-452.

Bauer, W. R., Crick, F. H. C. \& White. J. H. (1980) Supercoiled DNA. Scientific American 243, 10011 .

BeCKer, A. \& GoLD, M. (1978). Enzymatic breakage of cohesive end site of phage-lambda-terminase (Ter) reaction. Procedings of the National Academ! of Sciences of the Linised States of Anterica 75, 4199 4203.

Brammar, W. J., Murray, N. F. \& Winton, S. (1974). Restriction of irp bacteriophages by Escherichia coli K. Journal of Molecular Biology 90. 63.3 647.

Echols, H. \& Murialdo, H. (1978). Genetic map of bacteriophage lambda. Microhiological Retiews 42. 577591.

ExQlist. L. W. \& Skalka. A. (1973). Replicilion of bacteriophage $i$ DNA dependent on the function of host and viral genes. Jourmal of Afolecular Biology 75. 185212.

Fiandt, M., Hradecha, Z., Lozeron, H. A. \& Szybalski. W. (1971). Electron microscope mapping of deletions. insertions, inversions and homologies in the DNAs of coliphages lambda and phi80. In The Bacteriophage Lambda. pp. 329 354. Edited by A. D. Hershey. New York: Cold Spring Harbor Laboratory

FraNkLIN, N. C. (1971). The $N$ operon of lambda extent and regulation as observed in fusions to the tryptophan operon of Escherichia coli. In The Bacteriophage Lambila. pp. 621 638. Fdited by A. D Hershey. Neu York: Cold Spring Harbor Laboratory.

Greer. H. (1975a). The kil gene of bacteriophage lambda. I'irolog. 66. 589 614.

Greer. H. (1975h). Host mutants resistant to phage lambda killing. I'irolog! 66. 605 609.

(jReER. H. \& Alstbel. F. (1979). Radiochemicial identification of the kil gene product of bacteriorphage i. Jirologl 95. 577580 .

Goldberg. A. R. \& Howe. M. (1969). New mutations in the $S$ cistron of bacteriophage lambda affecting host cells lysis. Iirology 38. 200201.

Hadfield. $(19811)$. $\lambda$ phasmids. PhD thesis. Liviversity of Leicester.

hadfield, C.\& Brammar. W. J. (1984). A genetic analysis of defective plasmid formation by $N$. deficient phage $i$ chromosomes. Journal of General Microhiolog. 130, 2501 251?

HeNderson. D. H. \& WeIl. J. (1975). Recombinationdeficient deletions in bacteriophage $\lambda$ and their interaction with chi mutations. Genetics 79. 143174.

Hopkins. A. S. Mtrray. N. F. \& Brammar, W. J. (1976). Chatacterization of $\lambda$ irp-transducing bacteriophages made in titro. Journal of Molecular Biolog. 107. 549569
Jeffreys, A. J. \& Flavell, R. A. (1977). A physical map of the DNA regions flanking the rabbit $\beta$ globin gene. $C e / l 12,4294.39$.

Kalser. K. \& Mlrray, N. F. (1979). Physical characterization of the "Rac prophage" in E. coli K12. Molecular and General Genetics 175. 159-174.

K LeCKNeR, N.\& SigNer. F. (1977). Genetic characterization of plasmid formation by $\boldsymbol{N}^{-}$mutants of bacteriophage $\lambda$. Virolog! 79. 160173.

LIEDKE-KCLKE, M. \& KAISER, A. D. (1967). The cregion of coliphage 21. l'irology 32. 475481 .

MCMilis. K. D. Stahl, M. M. \& Stahl. F. W (1974). Rec-mediated recombinational hot spot activity in bacteriophage lambda. I. Hot spot activity associated with $\mathrm{Spi}^{-}$deletions and hio substitutions. Genesics 77, 409423

Meselsox. M. (1964). On the mechanism of genetic recombination between DNA molecules. Jourrul of Molecular Biolog! 9. 7.34 745.

Mirray, K., Murray, N. E. \& Bertani, G. (1975). Base changes in the recognition site for ter functions in lambdoid phage DNA. Nature. London 254. 262 265.

Mlrray. N. F. \& Brammar, W. J. (1973). The irpE gene of Escherchia coli $\mathbf{K}$ contains a recognition sequence for the K-restriction system. Journal of Molecular Biolog! 77. 615624 .

mirray, N. F., Brammar, W. J. \& Mtrray. K. (1977). Lambdoid phages that simplify the recovery of in ritro recombinants. Molecular and General Ge'metics 150, 53 61

Pirrotta. V., Hadfield, C. \& Pretorjls. G. H. (1983). Microdissection and cloning of the white locus and the $3 \mathrm{~B} /-3 \mathrm{C} 2$ region of the Drosuphila $\mathrm{X}$ chromosome. EMBO Journal 2, 9279.34.

ReAder. R. W. \& Siminovitch. L. (1971a). Lysis defective mutants of bacteriophage lambda: genetics and physiology of $S$ cistron mutants. Virology 43.607 622.

ReAder. R. W. \& Siminovitch. L. (197/h). Lysis defective mutants of bacteriophage lambda : on the role of the $S$ function in lysis. Firology 43.623 637 .

Sanger. F. Collson, A. R., HoNg, G. F., Hill, D. F. \& Petersen, G. B. (1982). Nucleotide sequence of bacteriophage ; DNA. Journal of Molecular Biolog. 162. 729774 .

Signer. F. (1969). Plasmid formation: a new mode of lysogeny by phage $\lambda$. Nature. London 223. 150168.

Smith, G. F. \& SLMmers, $M$. D. (1980). The bidirectional transfer of DNA and RNA to nitrocellulose or diazobenzyloxymethyl-paper. Analifical Biochemistry 109, 123129.

SLMNER-SMITH, M. \& BeCKer. A. (1981). DNA packaging in the lambdoid phages: jdentification of the products of $\phi 80$ genes I and 2. Firology 111,629 64I.

SimNer-Smith, M. \& Gold. M. (1981). DNA packaging in the lambdoid phages: the role of $\lambda$ genes Nul and .4. l'irolog! 111. 642646.

Szybalski. F. H. \& Szybalski. W. (1979). A comprehensive molecular map of bacteriophage lambda. Ge'm 7.217270.

WasG, J. (.. \& KaISER. A. D. (1973). Fvidence that the cohesive ends of mature $\lambda$ DNA are generaled by the gene $A$ product. Nature New Biologi 241. 1617. 


\section{HADFIELD ANDW. J. BRAMMAR}

Weil. J. \& Signer, E. R. (1968). Recombination in bacteriophage $\lambda$. Mutants deficient in general recombination. Journal of Molecular Biology 34, 261 271.

WITKIN, E. W. (1976). Ultraviolet mutagenesis and inducible DNA repair in Escherichia coli. Bacteriological Rerien's 40, 869-907.
Youderain, P. (1977). Genetic, physical and restriction map of bacteriophage $\phi 80$. In DNA Insertion Elements, Plasmids and Episomes, Pp. 74l-744. Edited by A. I. Bukari, J. A. Shapiro \& S. L. Adhya. New York: Cold Spring Harbor Laboratory. 